\title{
Chemical gradients in the Milky Way from the RAVE data
}

\section{Dwarf stars}

\author{
C. Boeche ${ }^{1,2}$, A. Siebert ${ }^{3}$, T. Piffl ${ }^{2}$, A. Just ${ }^{1}$, M. Steinmetz ${ }^{2}$, S. Sharma ${ }^{4}$, G. Kordopatis ${ }^{5}$, G. Gilmore ${ }^{5}$, C. Chiappini ${ }^{2}$,
} M. Williams ${ }^{2}$, E. K. Grebel ${ }^{1}$, J. Bland-Hawthorn ${ }^{4}$, B. K. Gibson ${ }^{6,7}$, U. Munari ${ }^{8}$, A. Siviero ${ }^{9,2}$, O. Bienaymé ${ }^{3}$, J. F. Navarro ${ }^{10}$, Q. A. Parker ${ }^{11,12,13}$, W. Reid ${ }^{12}$, G. M. Seabroke ${ }^{14}$, F. G. Watson ${ }^{13}$, R. F. G. Wyse ${ }^{15}$, and T. Zwitter ${ }^{16,17}$

1 Astronomisches Rechen-Institut, Zentrum für Astronomie der Universität Heidelberg, Mönchhofstr. 12-14, 69120 Heidelberg, Germany e-mail: corrado@ari.uni-heidelberg.de

2 Leibniz Institut für Astrophysik Potsdam (AIP), An der Sternwarte 16, 14482 Potsdam, Germany

3 Observatoire de Strasbourg, Université de Strasbourg, CNRS 11 rue de l’Université, 67000 Strasbourg, France

${ }^{4}$ Sydney Institute for Astronomy, School of Physics A28, University of Sydney, NSW 2006, Australia

5 Institute of Astronomy, University of Cambridge, Madingley Road, Cambridge CB3 OHA, UK

${ }^{6}$ Jeremiah Horrocks Institute, University of Central Lancashire, Preston, PR1 2HE, UK

7 Monash Centre for Astrophysics, School of Mathematical Sciences, Monash University, Clayton, VIC, 3800, Australia

8 INAF Osservatorio Astronomico di Padova, via dell’Osservatorio 8, 36012 Asiago, Italy

9 Department of Physics and Astronomy, Padova University, Vicolo dell'Osservatorio 2, 35122 Padova, Italy

${ }^{10}$ University of Victoria, PO Box 3055, Station CSC, Victoria, BC V8W 3P6, Canada

11 Department of Physics \& Astronomy, Macquarie University, NSW 2109 Sydney, Australia

12 Research Centre for Astronomy, Astrophysics and Astrophotonics, Macquarie University, NSW 2109 Sydney, Australia

13 Australian Astronomical Observatory, PO Box 915, NSW 1670 North Ryde, Australia

14 Mullard Space Science Laboratory, University College London, Holmbury St Mary, Dorking, RH5 6NT, UK

15 Department of Physics and Astronomy, Johns Hopkins University, 3400 North Charles Street, Baltimore, MD 21218, USA

${ }^{16}$ Faculty of Mathematics and Physics, University of Ljubljana, Jadranska 19, 1000 Ljubljana, Slovenia

17 Center of Excellence SPACE-SI, Askerceva cesta 12, 1000 Ljubljana, Slovenia

Received 14 June 2013 / Accepted 27 August 2013

\section{ABSTRACT}

\begin{abstract}
Aims. We aim at measuring the chemical gradients of the elements $\mathrm{Mg}, \mathrm{Al}, \mathrm{Si}$, and $\mathrm{Fe}$ along the Galactic radius to provide new constraints on the chemical evolution models of the Galaxy and Galaxy models such as the Besançon model. Thanks to the large number of stars of our RAVE sample we can study how the gradients vary as function of the distance from the Galactic plane.

Methods. We analysed three different samples selected from three independent datasets: a sample of 19962 dwarf stars selected from the RAVE database, a sample of 10616 dwarf stars selected from the Geneva-Copenhagen Survey (GCS) dataset, and a mock sample (equivalent to the RAVE sample) created by using the GALAXIA code, which is based on the Besançon model. The three samples were analysed by using the very same method for comparison purposes. We integrated the Galactic orbits and obtained the guiding radii $\left(R_{\mathrm{g}}\right)$ and the maximum distances from the Galactic plane reached by the stars along their orbits $\left(Z_{\max }\right)$. We measured the chemical gradients as functions of $R_{\mathrm{g}}$ at different $Z_{\max }$.

Results. We found that the chemical gradients of the RAVE and GCS samples are negative and show consistent trends, although they are not equal: at $Z_{\max }<0.4 \mathrm{kpc}$ and $4.5<R_{\mathrm{g}}(\mathrm{kpc})<9.5$, the iron gradient for the RAVE sample is d[Fe $\left./ \mathrm{H}\right] / \mathrm{d} R_{\mathrm{g}}=-0.065 \mathrm{dex} \mathrm{kpc}{ }^{-1}$, whereas for the GCS sample it is $\mathrm{d}[\mathrm{Fe} / \mathrm{H}] / \mathrm{d} R_{\mathrm{g}}=-0.043 \mathrm{dex} \mathrm{kpc}^{-1}$ with internal errors of \pm 0.002 and \pm 0.004 dex kpc ${ }^{-1}$, respectively. The gradients of the RAVE and GCS samples become flatter at larger $Z_{\max }$. Conversely, the mock sample has a positive iron gradient of $\mathrm{d}[\mathrm{Fe} / \mathrm{H}] / \mathrm{d} R_{\mathrm{g}}=+0.053 \pm 0.003 \mathrm{dex} \mathrm{kpc}^{-1}$ at $Z_{\max }<0.4 \mathrm{kpc}$ and remains positive at any $Z_{\max }$. These positive and unrealistic values originate from the lack of correlation between metallicity and tangential velocity in the Besançon model. In addition, the low metallicity and asymmetric drift of the thick disc causes a shift of the stars towards lower $R_{\mathrm{g}}$ and metallicity which, together with the thin-disc stars with a higher metallicity and $R_{\mathrm{g}}$, generates a fictitious positive gradient of the full sample. The flatter gradient at larger $Z_{\max }$ found in the RAVE and the GCS samples may therefore be due to the superposition of thin- and thick-disc stars, which mimicks a flatter or positive gradient. This does not exclude the possibility that the thick disc has no chemical gradient. The discrepancies between the observational samples and the mock sample can be reduced by i) decreasing the density; ii) decreasing the vertical velocity; and iii) increasing the metallicity of the thick disc in the Besançon model.
\end{abstract}

Key words. Galaxy: abundances - Galaxy: evolution - Galaxy: structure - Galaxy: kinematics and dynamics

\section{Introduction}

The spatial distribution of the chemical abundances in the Milky Way provides important constraints for our understanding of galaxy formation. The distribution of the chemical species is a tracer of the way the galaxies formed because the abundances in the stellar atmospheres of a stellar population do not change during its lifetime ${ }^{1}$, in contrast to its spatial distribution and kinematics. Stars inherit the metallicity of the interstellar

\footnotetext{
1 An exception to this occurs in the last stages of the stellar evolution, when the chemical abundances of the stellar atmosphere can be changed by dredge-ups, which bring the products of the nucleosynthesis to the surface.
} 
medium from which they form. Its metallicity depends in a complex way on the infall and outflow of gas and the enrichment by older stellar populations. Additionally, the element abundance ratios depend on the speed of enrichment. Fast enrichment results in an $\alpha$-enhancement because $\alpha$-elements are mainly produced by supernovae ( $\mathrm{SNe}$ ) type II on a short timescale, whereas most of the iron is produced by SN type Ia with a significant time delay. Therefore the abundances of stellar populations are tightly connected to the gas infall and star formation history. As a consequence, two locations that experienced different star formation histories exhibit a difference (gradient) in chemical abundances.

Today the existence of a negative chemical gradient along the Galactic radius is well established thanks to several dedicated observational works (see Maciel \& Costa 2010, and references therein). Chemical gradients were measured by means of several tracers (such as Cepheids, planetary nebulae, open clusters) that cover a large range of Galactocentric distances $(R)$. The chemical gradients found by previous investigations can span

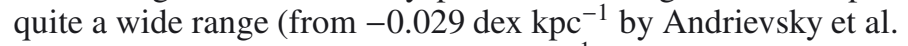
2002 using Cepheids, to $-0.17 \mathrm{dex} \mathrm{kpc}^{-1}$ by Sestito et al. 2008 using open clusters). Most of the works seem to converge on

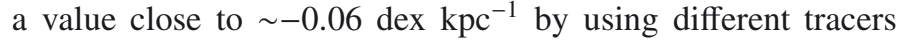
(Cepheids by Luck et al. 2006; Luck \& Lambert 2011; Yong et al. 2006; Lemasle et al. 2008 among others; open clusters by Friel et al. 2002; Sestito et al. 2008; Pancino et al. 2010 among others; planetary nebulae by Pasquali \& Perinotto 1993; Maciel \& Quireza 1999; main-sequence turn-off stars by Cheng et al. 2012; Coşkunoğlu et al. 2012). It has been shown that the radial gradient becomes flatter with increasing distance from the Galactic plane (Pasquali \& Perinotto 1993; Boeche 2011; Cheng et al. 2012). If this flattening is caused by a zero radial gradient in the thick disc (Allende Prieto et al. 2006; Ruchti et al. 2011; Bilir et al. 2012) or an effect of a flattening agent such as radial mixing (as dynamical and chemo-dynamical models would suggest, see Sellwood \& Binney 2002; Schönrich \& Binney 2009a; Sánchez-Blázquez et al. 2009; Minchev et al. 2011, 2013) and energy feedback (Gibson et al. 2013) is still unclear.

To explain the gradients observed in the Milky Way, an inside-out formation scenario of the Milky Way has been proposed (Matteucci \& François 1989) in which the inner parts of the Galaxy experienced a higher star formation rate than the outer parts. Galactochemical models (Chiappini et al. 1997; Cescutti et al. 2007) and hydrodynamical simulations (Pilkington et al. 2012) with inside-out formation show radial gradients consistent with the observed one. The study by Schönrich \& Binney (2009a) claims that there is no need to postulate an inside-out star formation to obtain negative gradients. In their model the whole disc forms simultaneously, and a metallicity gradient of $\sim-0.11 \mathrm{dex} \mathrm{kpc}^{-1}$ is finally obtained because of the advection inwards of heavier elements released by SNe. Observational data (see Sestito et al. 2008; Luck et al. 2006; Carraro et al. 2007; Yong et al. 2012 among others) suggest that there are variations of the gradient along $R$ : a steeper gradient in the inner disc $(R \lesssim 5 \mathrm{kpc})$ and a flatter gradient in the outer disc $(R \gtrsim 10 \mathrm{kpc})$.

In this paper we make use of the RAVE data to investigate the radial abundance gradients of the Milky Way. RAVE is a large spectroscopic survey that collected 574630 spectra of 483330 stars of the Milky Way. The spectra are centred on the near-infrared Ca II triplet window (8410-8795 $\AA$ ). The resolution of $R \simeq 7500$ allows measuring radial velocities (Steinmetz et al. 2006), stellar parameters (Zwitter et al. 2008; Siebert et al. 2011a; Kordopatis et al. 2013), and chemical abundances (Boeche et al. 2011; Kordopatis et al. 2013). Distance estimates (Breddels et al. 2010; Zwitter et al. 2010; Burnett et al. 2011; Binney et al. 2013) enable one to locate the stars in three-dimensional space. Proper motions are listed in a variety of catalogues: Tycho2 (Høg et al. 2000), the PPM-Extended catalogues PPMX and PPMXL (Roeser et al. 2008, 2010), and the second and third US Naval Observatory CCD Astrograph Catalog UCAC2 and UCAC3 (Zacharias et al. 2004). Chemical abundances for six elements are provided by the RAVE chemical pipeline (Boeche et al. 2011; Kordopatis et al. 2013), which delivers abundances with errors of $\sim 0.10-0.35$ dex, depending on the element, stellar parameters, and signal-to-noise ratio $(\mathrm{S} / \mathrm{N})$. The full set of kinematic and chemical data is a rich source of information for studying the Galactic disc and has been recently employed for several studies. By using the RAVE space and velocity data set, Siebert et al. (2011b) found a gradient in the mean Galactocentric radial velocity of stars in the extended solar neighbourhood, which has been confirmed by a recent work of Williams et al. (2013) who, using red-clump giant stars, found that it is more marked in the southern part of the disc, and furthermore highlights wave-like structures in the disc from the velocity distributions of the sample. The structure of the disk was studied by Pasetto et al. (2012a,b); Antoja et al. (2012), Wilson et al. (2011), and Veltz et al. (2008). The full set of RAVE chemo-kinematical data was recently used by Boeche et al. (2013) to study the relation between abundances and kinematics of the Galactic disc populations. The radial metallicity gradient was previously studied in recent works by using RAVE data (Karataş \& Klement 2012; Coşkunoğlu et al. 2012), but none of them investigated the gradients for the individual elemental abundances. Recently, Bilir et al. (2012) split a sample of red-clump RAVE stars into thin- and thick-disc subsamples by labelling the stars as a function of their kinematic characteristics, and measured the metallicity gradients in these subsamples.

In the present work we investigate the chemical gradients of four elements over the Galactocentric distance range of $4.5-9.5 \mathrm{kpc}$ by using the chemical and kinematic data of dwarf stars in the RAVE database. We also investigate how the gradients change as function of the distance from the Galactic plane. We applied the same analysis to the Geneva-Copenhagen Survey (GCS) data set (Nordström et al. 2004) with the recent data refinement by Casagrande et al. (2011) and with a mock sample constructed with the code GALAXIA (Sharma et al. 2011) to test the robustness of our results and compare them with Galactic models. We discuss how different stellar populations in the sample can generate a bias and affect the gradients measurements. This paper will be followed by a second paper in which we study the chemical gradients by using RAVE giant stars.

The paper is structured as follows: Sect. 2 describes the data and the selection of the star samples used to measure the gradients, in Sect. 3 we explain the analysis method applied to the samples, in Sects. 4 and 5 we report the chemical gradients obtained, in Sect. 6 we discuss biases and results and we conclude in Sect. 7.

\section{Data}

\subsection{RAVE sample}

We selected the sample from the RAVE internal data archive, which contains stellar atmospheric parameters $\left(T_{\text {eff }}\right.$, $\log g$ and $[\mathrm{M} / \mathrm{H}])$ of 451413 spectra, and chemical elemental abundances for 450641 spectra. The stellar atmospheric parameters are measured with the recently adopted 
RAVE pipeline (Kordopatis et al. 2011a, 2013). The chemical abundances $([\mathrm{X} / \mathrm{H}])$ for $\mathrm{Fe}, \mathrm{Al}, \mathrm{Mg}, \mathrm{Si}, \mathrm{Ti}$, and $\mathrm{Ni}$ are derived by the RAVE chemical pipeline (Boeche et al. 2011; Kordopatis et al. 2013). We adopted the parallaxes by Binney et al. (2013) computed with an improved version of the recipe described in Burnett et al. (2011).

With these data we determined Galactocentric positions and absolute velocities. Galactic orbital parameters such as the guiding radius $\left(R_{\mathrm{g}}\right)$ and maximum distance from the Galactic plane $\left(Z_{\max }\right)$ were considered and computed as explained in Sect. 3.1. In this first paper we limit our investigation to dwarf stars with good-quality RAVE spectra (i.e. good stellar parameters and chemical abundances) and reliable distances. The RAVE chemical catalogue provides abundances with errors $\sigma \sim 0.2$ dex for spectra with an $S / N>40$. These errors hold for the elements $\mathrm{Fe}, \mathrm{Mg}, \mathrm{Al}$, and $\mathrm{Si}$ ( $\mathrm{Ti}$ and Ni measurements have low precision for dwarf stars). Therefore, our selection criteria were i) stars with spectra with an $S / N>40$; ii) dwarf stars with gravity $\log g>3.8$ dex and effective temperatures $T_{\text {eff }}$ from 5250 to $7000 \mathrm{~K}$; and iii) stars with distance uncertainties smaller than $30 \%$. Moreover, we considered only the four elements Fe, $\mathrm{Mg}, \mathrm{Al}$, and Si because of their good precision $(\sigma \sim 0.15 \mathrm{dex})$ within the selected $T_{\text {eff }}$ and $\mathrm{S} / \mathrm{N}$ ranges. We took care that no peculiar stars were included in the sample by selecting only spectra flagged as normal stars by Matijevič et al. (2012).

Among these stars, we furthermore selected only spectra for which the RAVE pipeline converged to a single point of the parameter space (Algo_Conv parameter equal to zero). These spectra are well fit by the reconstructed spectra of the RAVE chemical pipeline $\left(\chi^{2}<2000\right)$, and with minor or no continuum $\operatorname{defects}^{2}$ (frac $>0.7$ ). Since RAVE re-observed $\sim 10 \%$ of the objects, some stars have two or more spectroscopic observations. To avoid multiple observations in our sample, we chose only the spectrum with the highest $\mathrm{S} / \mathrm{N}$ for re-observed stars. This selection yielded 19962 stars, whose spatial distribution is shown in Fig. 1. Most of the stars lie within $0.3 \mathrm{kpc}$ from the Galactic plane and in the Galactic radius interval $R=7.6-8.3 \mathrm{kpc}$. This is a small interval where we can estimate the local gradient, although its small spatial coverage does not allow precise measurements. We extended the gradient measurements to the Galactic radius range $R \sim 4.5-9.5 \mathrm{kpc}$ by using the guiding radius $R_{\mathrm{g}}$.

\subsection{Geneva-Copenhagen Survey sample}

The GCS provides information such as the temperature, metallicity, kinematics, distance, and age of $16682 \mathrm{~F}$ and $\mathrm{G}$ dwarf stars in the solar neighbourhood (Nordström et al. 2004). We here refer to the more recent values reported by Casagrande et al. (2011). We selected stars by using the same constraints in $T_{\text {eff }}$, $\log g$, and guiding radii $R_{\mathrm{g}}$ as were used for RAVE stars to keep the characteristics of the GCS and RAVE samples as similar as possible. With these constraints we selected 10616 stars. Still, there are some differences. In fact, GCS stars are spherically distributed in space and most of them do not lie farther away than $0.2 \mathrm{kpc}$ from the Galactic plane, whereas the RAVE stars have a cone-shaped distribution in space and extend up to $0.5 \mathrm{kpc}$ from the Galactic plane (Fig. 1). Because most of the RAVE sample is inside the scale height of the thin disc $(\sim 0.3 \mathrm{kpc})$, it is reasonable to assume that the difference in stellar populations between the

\footnotetext{
2 The frac parameter described in Boeche et al. (2011) gives the fraction of pixels that are non-defective and represents the goodness of the continuum fitting.
}
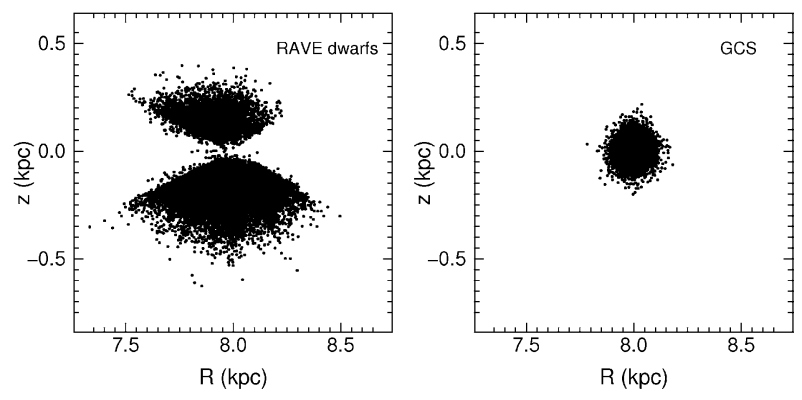

Fig. 1. Spatial distribution of the selected 19962 RAVE stars (left $)$ and 10616 stars of the GCS (right) on the $(R, z)$.

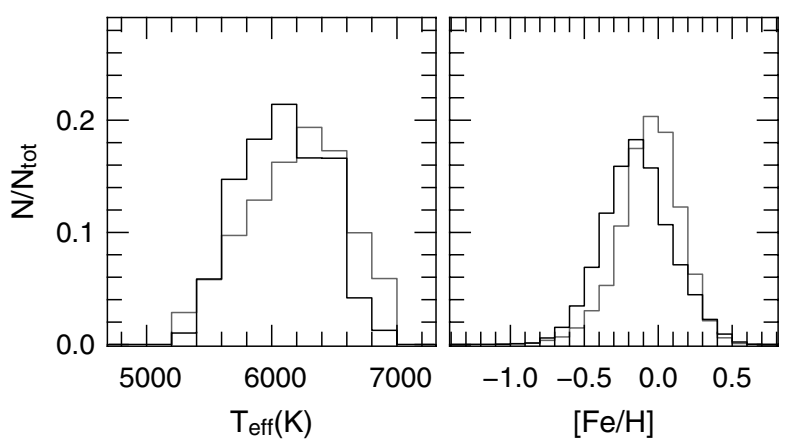

Fig. 2. Temperature (left) and metallicity (right) distributions of the RAVE (black line) and GCS (grey line) samples.

two samples is small. We expect a slight underrepresentation of the young, dynamically cold sub population in the RAVE sample compared with the GCS sample because of the cone structure of the RAVE volume. In Fig. 2 we compare the $T_{\text {eff }}$ and $[\mathrm{Fe} / \mathrm{H}]$ distributions of the two samples. The GCS sample has a temperature distribution favouring higher $T_{\text {eff }}$ than the RAVE sample, and the $[\mathrm{Fe} / \mathrm{H}]$ distribution is shifted by $\sim 0.1$ dex towards higher metallicities. Despite the highlighted differences, the GCS sample shows slightly flatter chemical gradients (but still in reasonable agreement) than the RAVE sample, as we will see below.

\subsection{RAVE mock sample}

The magnitude and spatial distributions of the RAVE sample may generate observational biases that can lead to misinterpretations. To avoid this, we created an equivalent mock sample with the stellar population synthesis code GALAXIA (Sharma et al. 2011), which uses analytical density profiles based on the Besançon model (Robin et al. 2003). The mock sample reproduces the detailed RAVE selection function in $I$ magnitude and target density, both as functions of angular position on the $\mathrm{sky}^{3}$. Only RAVE spectra with $S / N>40$ and flagged as normal stars by Matijevič et al. (2012) were considered.

The resulting mock catalog agrees well with the original RAVE catalog for the stellar parameters and the distance and radial velocity distributions. From this sample of 97485 entries, we imposed the same selection criteria in $T_{\text {eff }}$ and $\log g$ to mimic our RAVE sample, obtaining a mock sample of 26198 entries. The higher number of stars in the mock sample than the RAVE sample is due to other selection criteria, that are applied to the RAVE sample (such as Algo_Conv parameter convergence, $\chi^{2}$, frac parameters and a distance estimate) and not to the

\footnotetext{
The statistics on the celestial sphere were derived with the HEALPix package (Górski et al. 2005).
} 
underlying Galaxy model. The selected cut in $\mathrm{S} / \mathrm{N}(S / N>40)$ helps minimising these differences, which should not affect the results on the abundance gradients significantly. In fact, although the spectrum quality parameters change as a function of the distance (because RAVE is a magnitude-limited survey with constant integration time, more distant stars have on average lower $\mathrm{S} / \mathrm{N}$ spectra), this will equally affect stars at any Galactocentric distance. However, to stay on the safe side, we verified that the results were consistent with those obtained by applying the cut when no cuts in distance error were applied to the RAVE data.

\section{Method and error estimation}

\subsection{Galactic orbit integration}

Because stars are oscillating radially and vertically along their orbits, stellar samples in the solar neighbourhood contain information for a much larger volume. To take advantage of this, we integrated the Galactic orbits for a time of $40 \mathrm{Gyr}$ (corresponding to more than 50 periods for most of the stars) with the code NEMO (Teuben et al. 1995). As Galactic potential we adopted the potential model n. 2 by Dehnen \& Binney (1998), which assumes $R_{\odot}=8.0 \mathrm{kpc}$, and best matches the observed properties of the Galaxy. We used the same potential for all samples, including the mock sample based on the Besançon model. In axisymmetric equilibrium models the z-component of the angular momentum $L z$ and the vertical action $J z$ are constants of motion. With the help of the rotation curve $v_{\mathrm{c}}\left(R_{\mathrm{g}}\right)$ the angular momentum can be converted into the guiding radius $R_{\mathrm{g}}$. The commonly used mean radius $R_{\mathrm{m}}$ of apocentre $R_{\mathrm{a}}$ and pericentre $R_{\mathrm{p}}$ is a proxy for the guiding radius only for low eccentricities, therefore $R_{\mathrm{g}}$ has to be preferred. With these quantities we can study the gradients at Galactic radii beyond the range currently occupied by the stars. Vertically, the longest distance to the Galactic plane $Z_{\max }$ is a measure of the vertical action.

The values $R_{\mathrm{g}}$ and $Z_{\max }$ were computed with the method just described for the RAVE, the GCS, and the mock samples.

\subsection{Gradient error estimation}

We measured the abundance gradients by fitting the distribution of the stars in the $\left(R_{\mathrm{g}},[\mathrm{X} / \mathrm{H}]\right)$ plane with a linear regression. We also investigated more sophisticated methods such as modelling the density distribution of the stars with a two-dimensional function such as a Gaussian or lognormal function, and performing the fitting procedure with the maximum-likelihood method. The different methods provided consistent results. The linear regression was ultimately preferred because it is simpler and faster, in particular during the confidence interval evaluation. The gradient confidence intervals reported in this paper were evaluated by using a resampling method such as bootstrapping. Means and $1 \sigma$ errors (68\% confidence) were computed over 1000 realizations for every gradient reported. These errors represent the internal errors, as explained in the following section.

\subsection{Bias in the gradients and gradient errors}

One needs to be aware that the use of the guiding radius $R_{\mathrm{g}}$ introduces a kinematic bias for volume-limited samples such as the RAVE and GCS samples. Stars with small $R_{\mathrm{g}}$ can reach the solar neighbourhood only if they move in eccentric (kinematically hot) orbits. Such stars are on average more metal-poor than stars at the same $R_{\mathrm{g}}$ and in circular orbits, which will never reach the solar neighbourhood. This causes a lack of metal-rich stars at small $R_{\mathrm{g}}$ in volume-limited samples, and for these samples the measured gradient is less negative. The lack of metal-rich stars occurs at large $R_{\mathrm{g}}$ as well, with the difference that in the outer Galaxy the stellar density is lower, therefore the effect is weaker. Although we cannot precisely quantify this bias, we discuss the impact of this bias on the measured gradients.

\section{Analysis and results}

In this section we split the samples into three ranges of $Z_{\max }$, discuss the $[\mathrm{Fe} / \mathrm{H}]$ distributions, and derive the radial gradients in $[\mathrm{Fe} / \mathrm{H}]$ and in $[\alpha / \mathrm{Fe}]$. In Sect. 5 we refine the method for the RAVE sample to improve the vertical resolution and extend the analysis to the elements $\mathrm{Fe}, \mathrm{Al}, \mathrm{Mg}$, and $\mathrm{Si}$ and the relative abundance gradients $[\mathrm{X} / \mathrm{Fe}]$.

\subsection{Gradients from the RAVE sample}

The resulting gradient for iron at the present position of the stars is $\frac{\mathrm{d}[\mathrm{Fe} / \mathrm{H}]}{\mathrm{d} R}=-0.029 \pm 0.011 \mathrm{dex} \mathrm{kpc}^{-1}$. The small range in $R$ covered by the sample $(7.6<R(\mathrm{kpc})<8.3)$ does not allow a precise measurement. The derived metallicity gradient is significantly flatter than expected, but this may be due to the conical volume, where a negative vertical gradient biases the radial gradient to lower values. Moreover, $R$ may not be representative of the distance at which the stars were born, since many of them do not have circular orbits. From the probability point of view, the guiding radius $R_{\mathrm{g}}$ better represents the Galactocentric distance of the star during one revolution around the Galactic centre. By using $R_{\mathrm{g}}$ and the constraints $4.5<R_{\mathrm{g}}(\mathrm{kpc})<9.5$ (to avoid the few outlier stars that can affect the estimation) we obtained the result

$$
\frac{\mathrm{d}[\mathrm{Fe} / \mathrm{H}]}{\mathrm{d} R_{\mathrm{g}}}=-0.059 \pm 0.002 \mathrm{dex} \mathrm{kpc}^{-1} \text {. }
$$

We found in a previous work (Boeche 2011) that different gradients are obtained at different $Z_{\max }$. We divided the full sample into three subsamples with different $Z_{\max }$ ranges: $0.0<Z_{\max }(\mathrm{kpc}) \leq 0.4$ (16456 stars, named $Z_{0.0}^{\text {RAVE }}$ sample) $0.4<Z_{\max }(\mathrm{kpc}) \leq 0.8$ (3032 stars, the $Z_{0.4}^{\mathrm{RAVE}}$ sample), and $Z_{\max }(\mathrm{kpc})>0.8$ (399 stars, the $Z_{0.8}^{\mathrm{RAVE}}$ sample). For these samples we found

$$
\begin{aligned}
& \frac{\mathrm{d}[\mathrm{Fe} / \mathrm{H}]}{\mathrm{d} R_{\mathrm{g}}}\left(Z_{0.0}^{\mathrm{RAVE}}\right)=-0.065 \pm 0.002 \mathrm{dex} \mathrm{kpc}^{-1} \\
& \frac{\mathrm{d}[\mathrm{Fe} / \mathrm{H}]}{\mathrm{d} R_{\mathrm{g}}}\left(Z_{0.4}^{\mathrm{RAVE}}\right)=-0.059 \pm 0.005 \mathrm{dex} \mathrm{kpc}^{-1} \\
& \frac{\mathrm{d}[\mathrm{Fe} / \mathrm{H}]}{\mathrm{d} R_{\mathrm{g}}}\left(Z_{0.8}^{\mathrm{RAVE}}\right)=0.005 \pm 0.012 \mathrm{dex} \mathrm{kpc}^{-1} .
\end{aligned}
$$

In Fig. 3 the gradients are represented by the slope of the straight line (left panels). We also report the gradients for $[\alpha / \mathrm{Fe}]^{4}$ :

$$
\begin{aligned}
& \frac{\mathrm{d}[\alpha / \mathrm{Fe}]}{\mathrm{d} R_{\mathrm{g}}}\left(Z_{0.0}^{\mathrm{RAVE}}\right)=-0.004 \pm 0.001 \mathrm{dex} \mathrm{kpc}^{-1}, \\
& \frac{\mathrm{d}[\alpha / \mathrm{Fe}]}{\mathrm{d} R_{\mathrm{g}}}\left(Z_{0.4}^{\mathrm{RAVE}}\right)=-0.005 \pm 0.002 \mathrm{dex} \mathrm{kpc}^{-1}, \\
& \frac{\mathrm{d}[\alpha / \mathrm{Fe}]}{\mathrm{d} R_{\mathrm{g}}}\left(Z_{0.8}^{\mathrm{RAVE}}\right)=-0.020 \pm 0.005 \mathrm{dex} \mathrm{kpc}^{-1} .
\end{aligned}
$$

\footnotetext{
4 We recall that in RAVE the $\alpha$-enhancement is computed as $[\alpha / \mathrm{Fe}]=\frac{[\mathrm{Mg} / \mathrm{Fe}]+[\mathrm{Si} / \mathrm{Fe}]}{2}$.
} 
RAVE

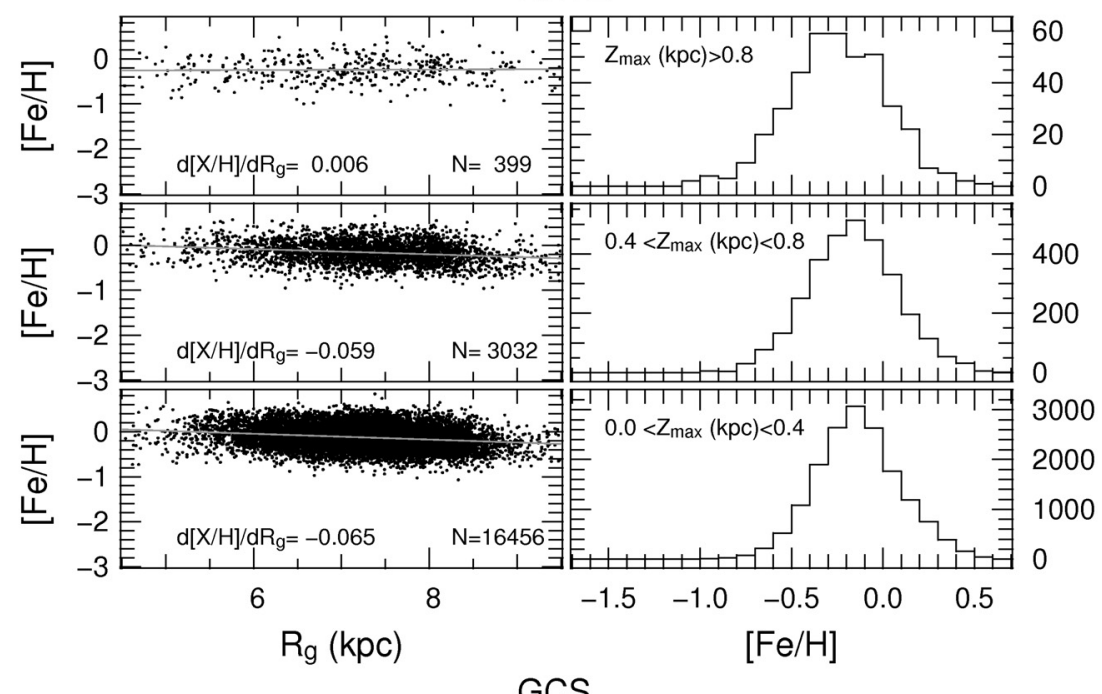

GCS

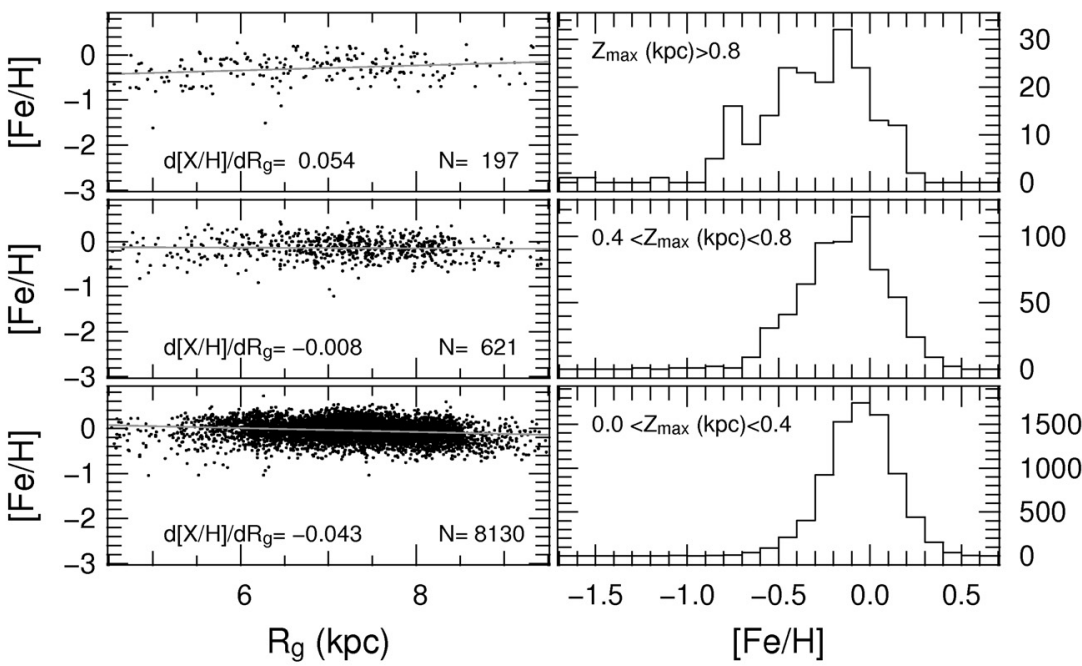

mock

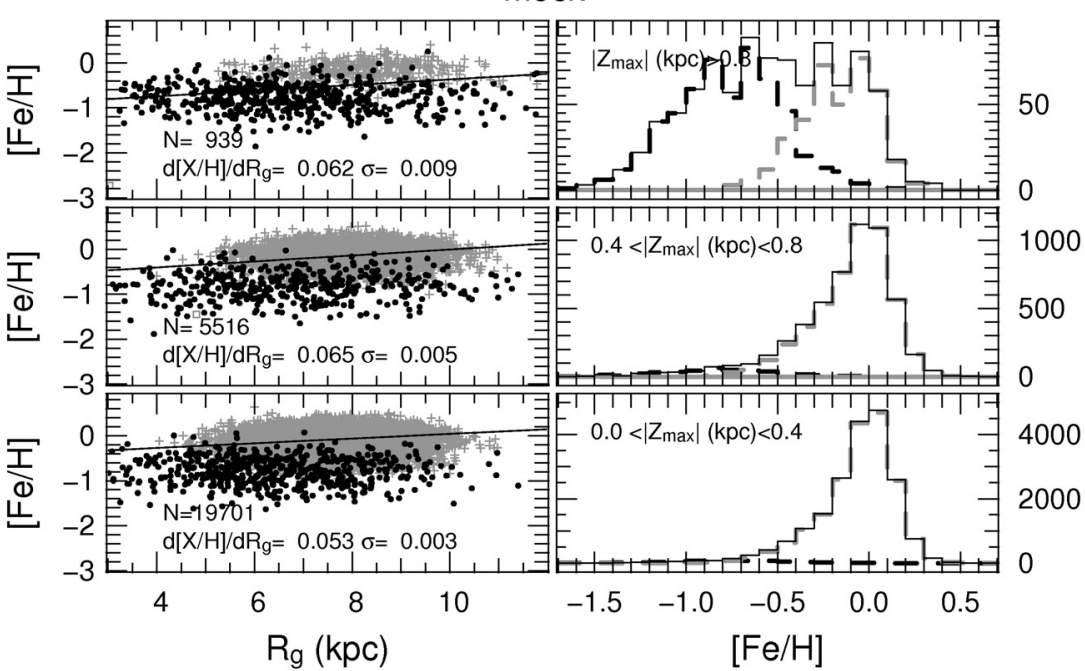

Fig. 3. Distributions of the dwarf stars for the RAVE sample (top), GCS sample (middle) and mock sample (bottom) in the ([Fe/H], $\left.R_{\mathrm{g}}\right)$ planes $(l e f t$ panels) and $[\mathrm{Fe} / \mathrm{H}]$ distributions (right panels). The grey line has a slope equal to the gradient. For the mock sample (bottom) grey plus symbols, black circles, and grey squares represent thin-disc, thick-disc, and halo stars, respectively. The same order is followed by the grey dashed, black dashed, and grey solid lines in the right panels, which represent the iron distributions of these subsamples. 
Table 1. Radial abundance gradients measured in the RAVE sample for $\mathrm{Fe}, \mathrm{Mg}, \mathrm{Al}$, and $\mathrm{Si}$ expressed as dex kpc ${ }^{-1}$ for three ranges of $Z_{\mathrm{max}}$.

\begin{tabular}{lcccc}
\hline \hline & $\frac{\mathrm{d}[\mathrm{Fe} / \mathrm{H}]}{\mathrm{d} R_{\mathrm{g}}}$ & $\frac{\mathrm{d}[\mathrm{Mg} / \mathrm{H}]}{\mathrm{d} R_{\mathrm{g}}}$ & $\frac{\mathrm{d}[\mathrm{Al} / \mathrm{H}]}{\mathrm{d} R_{\mathrm{g}}}$ & $\frac{\mathrm{d}[\mathrm{Si} / \mathrm{H}]}{\mathrm{d} R_{\mathrm{g}}}$ \\
\hline $0.0 \leq Z_{\max }(\mathrm{kpc})<0.4$ & $-0.065 \pm 0.003$ & $-0.073 \pm 0.002$ & $-0.085 \pm 0.003$ & $-0.064 \pm 0.003$ \\
$0.4 \leq Z_{\max }(\mathrm{kpc})<0.8$ & $-0.059 \pm 0.006$ & $-0.061 \pm 0.005$ & $-0.076 \pm 0.007$ & $-0.063 \pm 0.005$ \\
$Z_{\max }(\mathrm{kpc})>0.8$ & $+0.006 \pm 0.015$ & $-0.026 \pm 0.011$ & $-0.017 \pm 0.016$ & $-0.008 \pm 0.013$ \\
\hline
\end{tabular}

Notes. Uncertainties of $68 \%$ confidence are obtained with the bootstrap method.

Table 2. As Table 1, but for relative abundances $[\mathrm{X} / \mathrm{Fe}]$.

\begin{tabular}{lccc}
\hline \hline & $\frac{\mathrm{d}[\mathrm{Mg} / \mathrm{Fe}]}{\mathrm{d} R_{g}}$ & $\frac{\mathrm{d}[\mathrm{Al} / \mathrm{Fe}]}{\mathrm{d} R_{g}}$ & $\frac{\mathrm{d}[\mathrm{Si} / \mathrm{Fe}]}{\mathrm{d} R_{g}}$ \\
\hline $0.0 \leq Z_{\max }(\mathrm{kpc})<0.4$ & $-0.009 \pm 0.002$ & $-0.022 \pm 0.002$ & $+0.001 \pm 0.001$ \\
$0.4 \leq Z_{\max }(\mathrm{kpc})<0.8$ & $-0.004 \pm 0.003$ & $-0.018 \pm 0.004$ & $-0.004 \pm 0.003$ \\
$Z_{\max }(\mathrm{kpc})>0.8$ & $-0.027 \pm 0.007$ & $-0.024 \pm 0.009$ & $-0.013 \pm 0.006$ \\
\hline
\end{tabular}

These results confirm that the radial gradients in the RAVE sample vary with increasing $Z_{\max }$. The complete set of measured gradients of abundances $[\mathrm{X} / \mathrm{H}]$ and relative abundance $[\mathrm{X} / \mathrm{Fe}]$ for the RAVE elements are given in Tables 1 and 2.

\subsection{Gradients from the Geneva-Copenhagen survey sample}

To evaluate the robustness of our results we repeated the same procedure for the GCS data. With the actual Galactocentric radius $R$ of all stars, the gradient is $\frac{\mathrm{d}[\mathrm{Fe} / \mathrm{H}]}{\mathrm{d} R}=-0.004 \pm$ $0.047 \mathrm{dex} \mathrm{kpc}^{-1}$, which is unreliable because the very small radial extent of the sample.

With the guiding radius in the range $R_{\mathrm{g}} \sim 4.5-9.5 \mathrm{kpc}$ we

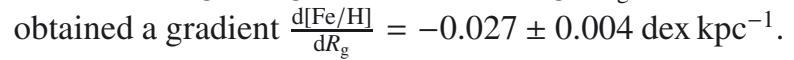

We followed the procedure used for the RAVE sample and selected three subsamples in the following $Z_{\max }$ ranges: $0.0<Z_{\max }(\mathrm{kpc})<0.4$ (8130 stars, named the $Z_{0.0}^{\mathrm{GCS}}$ sample) $0.4<Z_{\max }(\mathrm{kpc})<0.8$ (621 stars, the $Z_{0.4}^{\mathrm{GCS}}$ sample) and $Z_{\max }(\mathrm{kpc})>0.8\left(197\right.$ stars, the $Z_{0.8}^{\mathrm{GCS}}$ sample). The resulting gradients are (see also Fig. 3, middle)

$$
\begin{aligned}
& \frac{\mathrm{d}[\mathrm{Fe} / \mathrm{H}]}{\mathrm{d} R_{\mathrm{g}}}\left(Z_{0.0}^{\mathrm{GCS}}\right)=-0.043 \pm 0.004 \mathrm{dex} \mathrm{kpc}^{-1}, \\
& \frac{\mathrm{d}[\mathrm{Fe} / \mathrm{H}]}{\mathrm{d} R_{\mathrm{g}}}\left(Z_{0.4}^{\mathrm{GCS}}\right)=-0.008 \pm 0.011 \mathrm{dex} \mathrm{kpc}^{-1} \\
& \frac{\mathrm{d}[\mathrm{Fe} / \mathrm{H}]}{\mathrm{d} R_{\mathrm{g}}}\left(Z_{0.8}^{\mathrm{GCS}}\right)=+0.056 \pm 0.019 \mathrm{dex} \mathrm{kpc}-1
\end{aligned}
$$

The metallicity distribution shows a more densely populated low-metallicity tail at large $Z_{\max }$ than the RAVE sample. The gradients $\mathrm{d}[\mathrm{Fe} / \mathrm{H}] / \mathrm{d} R_{\mathrm{g}}$ are in general less negative than the RAVE sample. The trend of a flatter gradient with $Z_{\max }$ agrees with the RAVE data, with the exception of the $Z_{0.8}^{\mathrm{GCS}}$ subsample (which has a positive gradient), which is particularly affected by the bias discussed in Sect. 3.3. This gradient is probably not reliable, and in Sect. 6.2 we provide a more realistic value.

We tentatively measured the gradients of the $\alpha$-elements given by Casagrande et al. (2011), with the warning that these abundances were estimated from photometry and must be considered only a proxy of $[\alpha / \mathrm{Fe}]$. For $[\alpha / \mathrm{Fe}]$ we obtained

$$
\begin{aligned}
& \frac{\mathrm{d}[\alpha / \mathrm{Fe}]}{\mathrm{d} R_{\mathrm{g}}}\left(Z_{0.0}^{\mathrm{GCS}}\right)=+0.010 \pm 0.002 \mathrm{dex} \mathrm{kpc}^{-1}, \\
& \frac{\mathrm{d}[\alpha / \mathrm{Fe}]}{\mathrm{d} R_{\mathrm{g}}}\left(Z_{0.4}^{\mathrm{GCS}}\right)=-0.006 \pm 0.005 \mathrm{dex} \mathrm{kpc}^{-1}, \\
& \frac{\mathrm{d}[\alpha / \mathrm{Fe}]}{\mathrm{d} R_{\mathrm{g}}}\left(Z_{0.8}^{\mathrm{GCS}}\right)=-0.023 \pm 0.007 \mathrm{dex} \mathrm{kpc}^{-1} .
\end{aligned}
$$

Using only stars with more reliable temperature estimates (treated with the infrared flux method of Casagrande et al. 2010), the results do not change significantly (differences smaller than $1 \sigma)$.

\subsection{Gradients from the RAVE mock sample}

The mock sample allows us to separate the influence of the different components (thin disc, thick disc, stellar halo) on the properties of the RAVE sample. In Fig. 3 (bottom panel) the contribution of the three components are separately shown in every $Z_{\max }$ slice. The halo contribution is negligible, and the thick disc is detected as a prominent low-metallicity peak at $Z_{\max }>0.8 \mathrm{kpc}$. The Besançon model assumes a radial metallicity gradient of $\mathrm{d}[\mathrm{Fe} / \mathrm{H}] / \mathrm{d} R=-0.07 \mathrm{dex} \mathrm{kpc}^{-1}$ for the thin disc and no gradient in the thick disc. The gradients we found deviate significantly from these values for reasons that we analyse in Sect. 6.1. Using the actual galactocentric radius $R$, the gradient for the mock sample is $\frac{\mathrm{d}[\mathrm{Fe} / \mathrm{H}]}{\mathrm{d} R}=-0.156 \pm 0.010 \mathrm{dex} \mathrm{kpc}^{-1}$, significantly more negative than expected. This is probably caused by the uneven spatial coverage in RAVE.

Using the guiding radius $R_{\mathrm{g}}$, we obtained a gradient $\frac{\mathrm{d}[\mathrm{Fe} / \mathrm{H}]}{\mathrm{d} R_{\mathrm{g}}}=$ $+0.060 \pm 0.003 \mathrm{dex} \mathrm{kpc}^{-1}$.

Selecting three subsamples in the three different $Z_{\max }$ ranges as we did before, the resulting gradients are

$$
\begin{aligned}
& \frac{\mathrm{d}[\mathrm{Fe} / \mathrm{H}]}{\mathrm{d} R_{\mathrm{g}}}\left(Z_{0.0}^{\text {mock }}\right)=+0.053 \pm 0.003 \mathrm{dex} \mathrm{kpc}^{-1}, \\
& \frac{\mathrm{d}[\mathrm{Fe} / \mathrm{H}]}{\mathrm{d} R_{\mathrm{g}}}\left(Z_{0.4}^{\text {mock }}\right)=+0.065 \pm 0.005 \mathrm{dex} \mathrm{kpc}^{-1}, \\
& \frac{\mathrm{d}[\mathrm{Fe} / \mathrm{H}]}{\mathrm{d} R_{\mathrm{g}}}\left(Z_{0.8}^{\text {mock }}\right)=+0.063 \pm 0.008 \mathrm{dex} \mathrm{kpc}^{-1} .
\end{aligned}
$$

We were unable measure the $[\alpha / \mathrm{Fe}]$ gradient for the mock sample because the Besançon model does not provide this observable. 


\section{Gradient estimates with moving box car}

The previous results show that the gradient flattens when moving to higher $Z_{\max }$. Whether this change occurs smoothly or with a sudden transition can be investigated by measuring the gradient in an interval of constant width, centred on progressively larger distances from the plane.

We measured the gradient $\frac{\mathrm{d}[\mathrm{X} / \mathrm{H}]}{\mathrm{d} R_{\mathrm{g}}}$ of a sample of stars lying in the interval $\left[Z_{\max }-0.1, Z_{\max }+0.1\right]$ and moved this sampling interval by $0.1 \mathrm{kpc}$ steps from $0.1 \mathrm{kpc}$ towards larger $Z_{\max }$. To always have a statistically significant sample in these intervals, we imposed the condition that at every step the sample must contain no fewer than 200 stars. If there were fewer stars, the $Z_{\max }$ interval was extended until the condition was matched. Undersampling occurs in particular at $Z_{\max }>0.7 \mathrm{kpc}$, where the number of stars is small. We present the result in Fig. 4, where the gradient $\frac{\mathrm{d}[\mathrm{X} / \mathrm{H}]}{\mathrm{d} R_{\mathrm{g}}}$ is shown as a function of $Z_{\max }$. We found that the gradients remained constant for all elements until $Z_{\max } \sim 0.7 \mathrm{kpc}$, then they increase to shallower (or positive) gradients with a hint of a step occurring between 0.6 and $0.9 \mathrm{kpc}$. For $[\mathrm{Fe} / \mathrm{H}]$ the gradient of the RAVE sample is $-0.06 \mathrm{dex} \mathrm{kpc}^{-1}$ and $\sim 0$ at small and large $Z_{\max }$, respectively, slightly more negative than the GCS sample, where it amounts to -0.05 and $+0.02 \mathrm{dex} \mathrm{kpc}^{-1}$, respectively. The mock sample shows positive gradients at any $Z_{\max }$. The value of $+0.06 \mathrm{dex} \mathrm{kpc}^{-1}$ at low $Z_{\max }$ decreases to +0.02 at large $Z_{\max }$, where the thick disc dominates.

In Fig. 5 we show the abundance distributions $[\mathrm{X} / \mathrm{H}]$ as a function of $Z_{\max }$ for the four elements of the RAVE sample and for $[\mathrm{Fe} / \mathrm{H}]$ in the GCS and the mock sample. In this figure we allowed a minimum of 20 stars in the interval to see local changes in the mean abundance. There is a clear negative trend visible in all cases. However, the trend of $[\mathrm{Fe} / \mathrm{H}]$ for the GCS sample is slightly smoother but covers the same range. The mock sample behaves quite differently: although the mean iron abundance at small and large $Z_{\max }$ is similar to that of the RAVE and GCS samples $\left(\sim 0.6\right.$ dex difference between $Z_{\max }=0$ and $Z_{\max }=2 \mathrm{kpc}$ ), its evolution as a function of $Z_{\max }$ is quite different. While for RAVE and GCS the decrease of the mean Fe abundance in the range $0.0<Z_{\max }<1.6 \mathrm{kpc}$ appears to be approximately linear, for the mock sample it has a negative second derivative up to $Z_{\max } \sim 0.6 \mathrm{kpc}$ and becomes positive afterwards. This difference suggests that the transition between the thin and thick disc in RAVE and GCS differs from the prediction of the mock sample.

\section{Discussion}

An important goal of investigating the chemical gradients is to distinguish the effect of biases caused by the selection function, the impact of the thin disc/thick disc dichotomy, and the features of the intrinsic evolution of the thin disc by an inside-out growth (Matteucci \& François 1989). The three samples analysed here show some similarities, but also striking differences.

\subsection{Abundance gradients in the GALAXIA/Besançon mock sample}

In the local sample of any Milky Way model the spatial structure of components or sub populations are represented only indirectly and only if the model is set up in a dynamically consistent way. The radial scalelength of the density in the disc is represented by a proper choice of the asymmetric drift (the shift of the tangential velocity distribution with respect to the circular speed). Vertically, the thickness corresponds to the assigned velocity dispersion. In addition to the density distributions and kinematics, stellar metallicities are assigned to each subpopulation according to their current position in the Besançon model regardless of kinematics. The vertical metallicity distribution is set up in a consistent way, because each sub population is assigned a spatially homogeneous metallicity distribution. Radially, an uncorrelated local metallicity distribution would also require a homogeneous metallicity distribution. The additionally assigned spatial metallicity gradient of $\mathrm{d}[\mathrm{Fe} / \mathrm{H}] / \mathrm{d} R=-0.07 \mathrm{dex} \mathrm{kpc}^{-1}$ for the thin-disc sub populations would require the correlation of the metallicity with the tangential velocity or angular momentum to be dynamically consistent. This means that the local sample with no correlation of tangential velocity and metallicity looks like a sample of components with no radial metallicity gradient. This caveat could easily be overcome by assigning the metallicity distribution at each location as a function of the angular momentum (or tangential velocity), leading to a radial gradient with respect to $R_{\mathrm{g}}$. The radial gradient as a function of the current position $R$ would then be very similar (see e.g. Binney 2010 for a dynamical model).

Nevertheless, we can learn about the impact of a distinct thick disc with lower metallicity and higher velocity dispersion and stronger asymmetric drift on the mock sample. The thick disc with a mean metallicity of -0.78 dex and 0.3 dex internal dispersion has an asymmetric drift of $53 \mathrm{~km} \mathrm{~s}^{-1}$ and a tangential velocity dispersion of $51 \mathrm{~km} \mathrm{~s}^{-1}$ compared with 15 and $28 \mathrm{~km} \mathrm{~s}^{-1}$ for the old thin disc. This shifts the thick-disc stars to the lower left in the $\left(R_{\mathrm{g}},[\mathrm{Fe} / \mathrm{H}]\right)$ diagrams (Fig. 3 bottom) with respect to the thin-disc stars, generating an apparent decreasing mean $[\mathrm{Fe} / \mathrm{H}]$ with decreasing $R_{\mathrm{g}}$, or in other words, a fictitious positive gradient. This trend is stronger for larger $Z_{\max }$, because the fraction of thick-disc stars increases with a still dominating thin disc at large $R_{\mathrm{g}}$. For the analysis of thin disc properties the contamination by thick disc stars in the RAVE sample results in a bias towards less negative or even positive radial metallicity gradients with respect to $R_{\mathrm{g}}$.

An additional striking difference of the mock sample compared with the RAVE and the GCS samples is the large number of low-metallicity stars for $Z_{\max }>0.8 \mathrm{kpc}$ (see Fig. 3). The reasons for this deviation can be the i) thick-disc density; ii) its vertical velocity dispersion; and iii) its mean metallicity adopted by the Besançon model. A lower local density in the model (at present assumed to be $\sim 10 \%$ ) would reduce the peak proportionally, a lower vertical velocity dispersion (at present assumed to be $42 \mathrm{~km} \mathrm{~s}^{-1}$ ) would shift a significant part to the lower $Z_{\max }$ bin and slightly lift the low-metallicity tail, whereas a higher mean metallicity (at present assumed to be -0.78 dex) would merge the peak with the thin-disc distribution.

Previous authors have already shown that the thick disc might have a higher mean metallicity than the one assumed in the Besançon model. A thick-disc mean metallicity of $\sim-0.5$ dex was found by some authors (Bilir et al. 2012; Kordopatis et al. 2011b; Schlesinger et al. 2012; Soubiran et al. 2003) with a gradient that decreases the mean metallicity $\sim-0.8$ dex beyond $3 \mathrm{kpc}$ (Katz et al. 2011; Ivezić et al. 2008). The lack of a prominent low-metallicity tail in the RAVE and GCS samples compared with the mock sample supports these studies.

Despite the unrealistic gradients exhibited, the mock sample gives very precious information for data interpretation. It suggests that short scale-length metal-poor components (thick disc and halo stars) may accumulate at low $R_{\mathrm{g}}$, and, together with the thin-disc stars, cause a fictitious flatter or positive gradient 

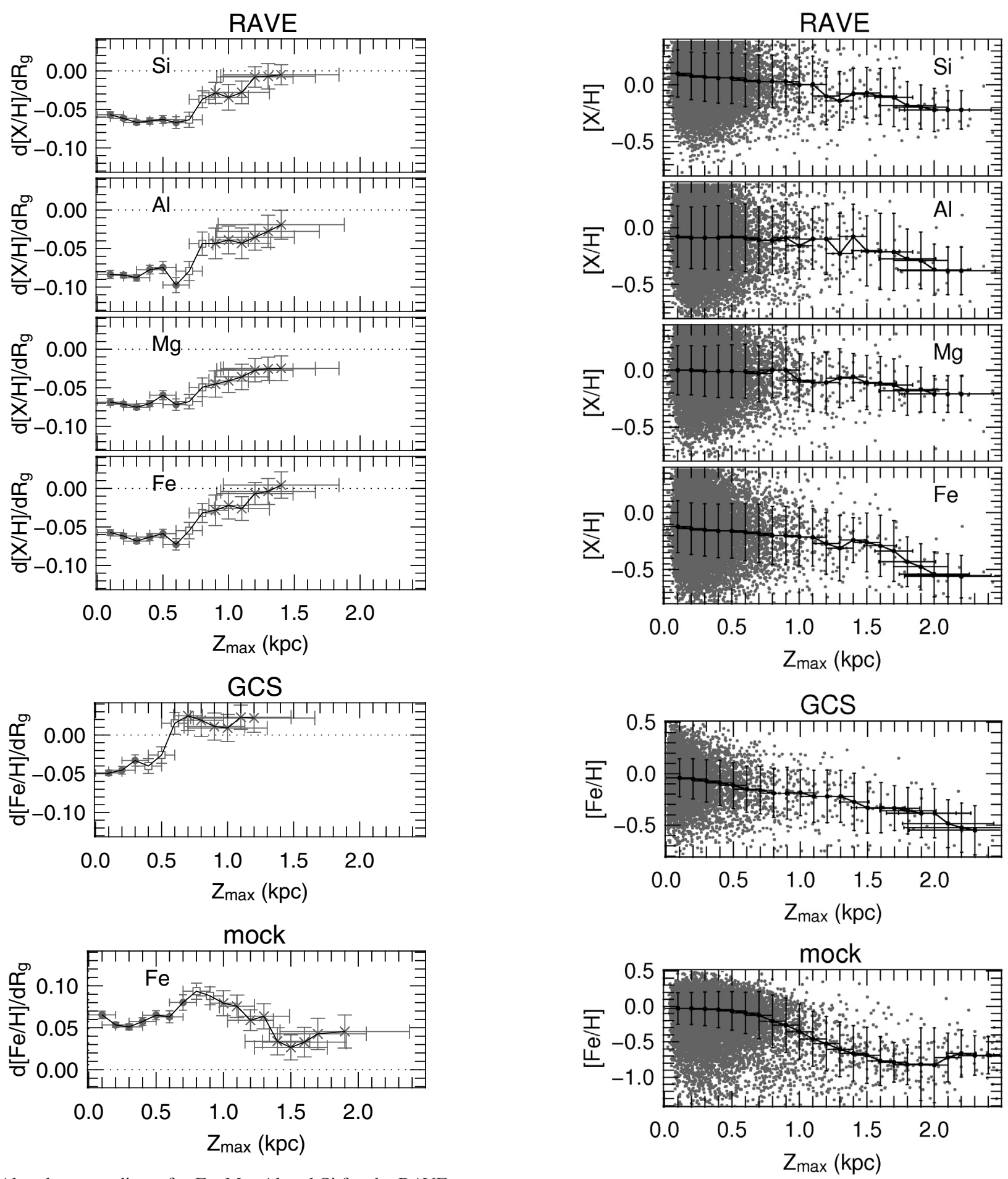

Fig. 4. Abundance gradients for $\mathrm{Fe}, \mathrm{Mg}, \mathrm{Al}$ and $\mathrm{Si}$ for the RAVE sample (top), $[\mathrm{Fe} / \mathrm{H}]$ for GCS sample (middle), and for the mock sample (bottom) as a function of $Z_{\max }$. Horizontal error bars indicate the $Z_{\max }$ interval in which the gradient has been measured. Vertical error bars indicate the gradient error estimated with the bootstrap technique outlined in Sect. 3.2. Full points indicate that the gradient has been computed with a sample containing $N>1000$ stars; open square represent samples with $1000<N<300$ stars; cross symbols represent samples with $200<N<300$ stars.

that depends on the relative fraction of the stellar populations in the sample. This might be one of the possible explanations of the positive metallicity gradients in thick-disc-like stars found in some previous works (Nordström et al. 2004; Carrell et al. 2012).

In observational data the population mix depends on the selection function of the sample under analysis, and only a complete (ideal) sample can deliver unbiased gradients. We must therefore be careful when interpreting the gradient measurement of any sample available today. 


\subsection{Abundance gradients of the observational samples}

In the previous section we saw how the mix of thin- and thickdisc stars in the Besançon model causes a positive gradient when the kinematically dependent quantity $R_{\mathrm{g}}$ is used. This effect is enhanced in our samples by the observational bias discussed in Sect. 3.3: metal-rich stars in (nearly) circular orbits at small $R_{\mathrm{g}}$ are missing because they cannot reach our volumelimited samples located in the solar neighbourhood, and the gradient becomes flatter than it should be. This bias is clearly visible in the GSC sample (Fig. 3, middle panel) where stars at $4.5 \leq R_{\mathrm{g}}(\mathrm{kpc}) \leq 5.5$ are clearly $[\mathrm{Fe} / \mathrm{H}]$ poorer than stars at higher $R_{\mathrm{g}}$. Excluding this $R_{\mathrm{g}}$ interval from the measurement, the gradients for the GCS data are $\mathrm{d}[\mathrm{Fe} / \mathrm{H}] / \mathrm{d} R_{\mathrm{g}}=-0.054 \pm$ $0.003 \mathrm{dex} \mathrm{kpc}-1, \quad-0.028 \pm 0.012 \mathrm{dex} \mathrm{kpc}^{-1}$, and $+0.004 \pm$ $0.025 \mathrm{dex} \mathrm{kpc}^{-1}$ for the subsamples $Z_{0.0}^{\mathrm{GCS}}, Z_{0.4}^{\mathrm{GCS}}$, and $Z_{0.8}^{\mathrm{GCS}}$, respectively, whereas for the RAVE data they are $\mathrm{d}[\mathrm{Fe} / \mathrm{H}] / \mathrm{d} R_{\mathrm{g}}=$ $-0.066 \pm 0.003 \mathrm{dex} \mathrm{kpc}^{-1},-0.063 \pm 0.006 \mathrm{dex} \mathrm{kpc}^{-1}$, and $-0.002 \pm 0.016 \mathrm{dex} \mathrm{kpc}^{-1}$ for the subsamples $Z_{0.0}^{\mathrm{RAVE}}, Z_{0.4}^{\mathrm{RAVE}}$, and $Z_{0.8}^{\text {RAVE }}$, respectively. The new GCS results are closer to those found with the RAVE data and, in particular, the peculiar positive gradient found with the $Z_{0.8}^{\mathrm{GCS}}$ sample disappears. We verified that adopting other cuts at low or high $R_{\mathrm{g}}$ causes changes in gradient of the order of 2-3 $\sigma$ for both the RAVE and the GCS samples (that means $\sim 0.01 \mathrm{dex} \mathrm{kpc}^{-1}$ ). These results suggest that the errors obtained with the bootstrap method (explained in Sect. 3.2) represent the internal errors, in which observational biases are neglected. Local inhomogeneities in the stellar population in the solar neighbourhood (such as disrupted open clusters and moving groups of kinematic origin, see Famaey et al. 2005; Antoja et al. 2012) can also explain the differences in gradient observed between the RAVE and the GCS samples and between different $R_{\mathrm{g}}$ intervals considered. Our conclusion is that these observational biases can affect the measured gradients for a quantity of about $\sim 0.01 \mathrm{dex} \mathrm{kpc}^{-1}$, which is larger than our internal errors, but it does not affect the interpretation of our results.

\subsection{Radial chemical gradients of the RAVE sample as a function of $Z_{\max }$}

In Sect. 4 we began the analysis by dividing the sample into three subsamples $Z_{0.0}^{\mathrm{RAVE}}, Z_{0.4}^{\mathrm{RAVE}}$, and $Z_{0.8}^{\mathrm{RAVE}}$. In the $R_{\mathrm{g}}$ interval $4.5-9.5 \mathrm{kpc}$, the $Z_{0.0}^{\mathrm{RAVE}}$ subsample exhibits a gradient of $\frac{\mathrm{d}[\mathrm{Fe} / \mathrm{H}]}{\mathrm{d} R_{m}} \sim-0.065 \mathrm{dex}$, which agrees with some of the works cited in Sect. 1. Moving to the subsamples $Z_{0.4}^{\mathrm{RAVE}}$ and $Z_{0.8}^{\mathrm{RAVE}}$, the gradients become less negative and then flatten, as also found in previous works (Cheng et al. 2012; Boeche 2011; Pasquali \& Perinotto 1993), but with these analyses it is not clear if the gradient changes smoothly or abruptly. Thanks to the large number of RAVE stars, we can use a moving box car to measure the radial gradients along $Z_{\max }$ (Fig. 4), and show that the gradients (of four different elements) are negative and remain approximately constant up to $Z_{\max } \sim 0.6 \mathrm{kpc}$, then they progressively increase to zero or positive values. In the light of the previous discussion of the Besançon model, the reasons for this change may be that i) the kinematically hot component(s) have no radial gradients; ii) the fraction of kinematically hot and metal poor component(s) increase and become significant at $Z_{\max }>0.6 \mathrm{kpc}$, generating a zero or positive gradient; or iii) both explanations are valid.

The information available up to this point does not allow us to decide whether the change of gradient at $Z_{\max } \sim 0.6 \mathrm{kpc}$ indicates the transition between one population (thin disc?) to another (thick disc?) or whether it is a mere artefact. If it were caused by a change from a population with a gradient (thin disc) to another one without a gradient (old thin disc or thick disc?) we would also expect a change in mean abundances at the same $Z_{\max }$. Figure 5 shows that the abundances gently diminish up to $Z_{\max } \sim 1.5 \mathrm{kpc}$ from the plane. Then, the slope becomes steeper until $Z_{\max } \sim 1.8 \mathrm{kpc}$, where the abundances become constant and where one would expect the thick disc to become dominant (but be aware of the poor statistic between 1.5 and $1.8 \mathrm{kpc}$ ). The GCS sample (Figs. 4 and 5) exhibits a similar pattern but at different $Z_{\max }$ : the rise to a flatter gradient occurs between 0.5 and $0.6 \mathrm{kpc}$ and a change of slope in iron abundance at $Z_{\max }>2.0 \mathrm{kpc}$. The change of slope at $Z_{\max } \sim 0.6 \mathrm{kpc}$ for the gradients and at $Z_{\max } \sim 1.5 \mathrm{kpc}$ for the mean abundance is imitated by the abundance enhancement $[\mathrm{X} / \mathrm{Fe}]$ shown in Figs. 6 and 7. The stars at $Z_{\max }<0.6 \mathrm{kpc}$ and $Z_{\max }>1.5 \mathrm{kpc}$ can be identified as thin- and thick-disc stars, respectively, while the stars in the middle region might be both the kinematically hot (old) part of the thin disc or a mix of thinand thick-disc stars. The absence of a clear step in abundances at $Z_{\max } \sim 0.6 \mathrm{kpc}$ excludes the transition between two populations at that height and supports the hypothesis that the change in gradients at $Z_{\max } \sim 0.6 \mathrm{kpc}$ is caused by metal-poorer, inner disc (thick disc?) stars, which creates the fictitious flatter or positive gradient discussed in Sect. 6.1. This does not exclude the presence of an old thin disk population or the absence of a gradient in the thick-disk population reported by some authors (Allende Prieto et al. 2006; Bilir et al. 2012).

\subsection{Observed radial chemical gradients of the thin disc (the $Z_{0.0}^{\text {RAVE }}$ sample)}

In Sect. 6.1 we discussed that a mix of different stellar populations can bias the measured gradients. In our data, the stars close to the Galactic plane are the less affected by this bias. This is confirmed in Fig. 4, which shows that the gradients remain constant up to $Z_{\max } \sim 0.6 \mathrm{kpc}$ because the number of thin-disc stars is larger than any other stellar populations that might be present in this region. The $Z_{0.0}^{\mathrm{RAVE}}$ sample is therefore more suitable for analysing and evaluating the measured chemical gradients in the framework of the star formation history of the Galactic disc.

In the Galactic radius interval $4.5-9.5 \mathrm{kpc}$, the $Z_{0.0}^{\mathrm{RAVE}}$ sample exhibits iron gradients of $\mathrm{d}[\mathrm{Fe} / \mathrm{H}] / \mathrm{d} R_{g}=-0.065 \mathrm{dex} \mathrm{kpc}{ }^{-1}$, which lies in the range covered by many observational studies cited in Sect. 1. The alpha elements $\mathrm{Mg}$ and $\mathrm{Si}$ exhibit similar gradients. Aluminium has a particularly negative gradient that distinguishes it from the other elements, and we briefly discuss it below.

Under the assumption of negligible gas infall, the abundance $[\mathrm{X} / \mathrm{H}]$ is a monotonically growing function of the number of $\mathrm{SNe}$; this means that a negative abundance gradient implies a higher number of $\mathrm{SNe}$ that exploded in the inner disc than the outer disc in relation to the gas reservoir. According to this simple remark, chemical evolution models of the Galaxy with a process of inside-out formation of the disc were developed (Chiappini et al. 1997; 2001; Cescutti et al. 2007; Gibson et al. 2013). Colavitti et al. (2009) showed that an inside-out formation of the disc is a necessary condition to explain the observed metallicity gradient. They predict negative abundance gradients that are slightly flatter than our values. For instance, Cescutti et al. found gradients of $-0.052,-0.039$ and $-0.035 \mathrm{dex} \mathrm{kpc}^{-1}$ for $\mathrm{Fe}, \mathrm{Mg}$, and $\mathrm{Si}$, respectively. For comparison, ours are -0.065 , -0.073 and -0.064 (see Table 1). Chiappini et al. 2001 reported a slightly negative gradient in $[\alpha / \mathrm{Fe}]$ in the range $4-8 \mathrm{kpc}$, which 

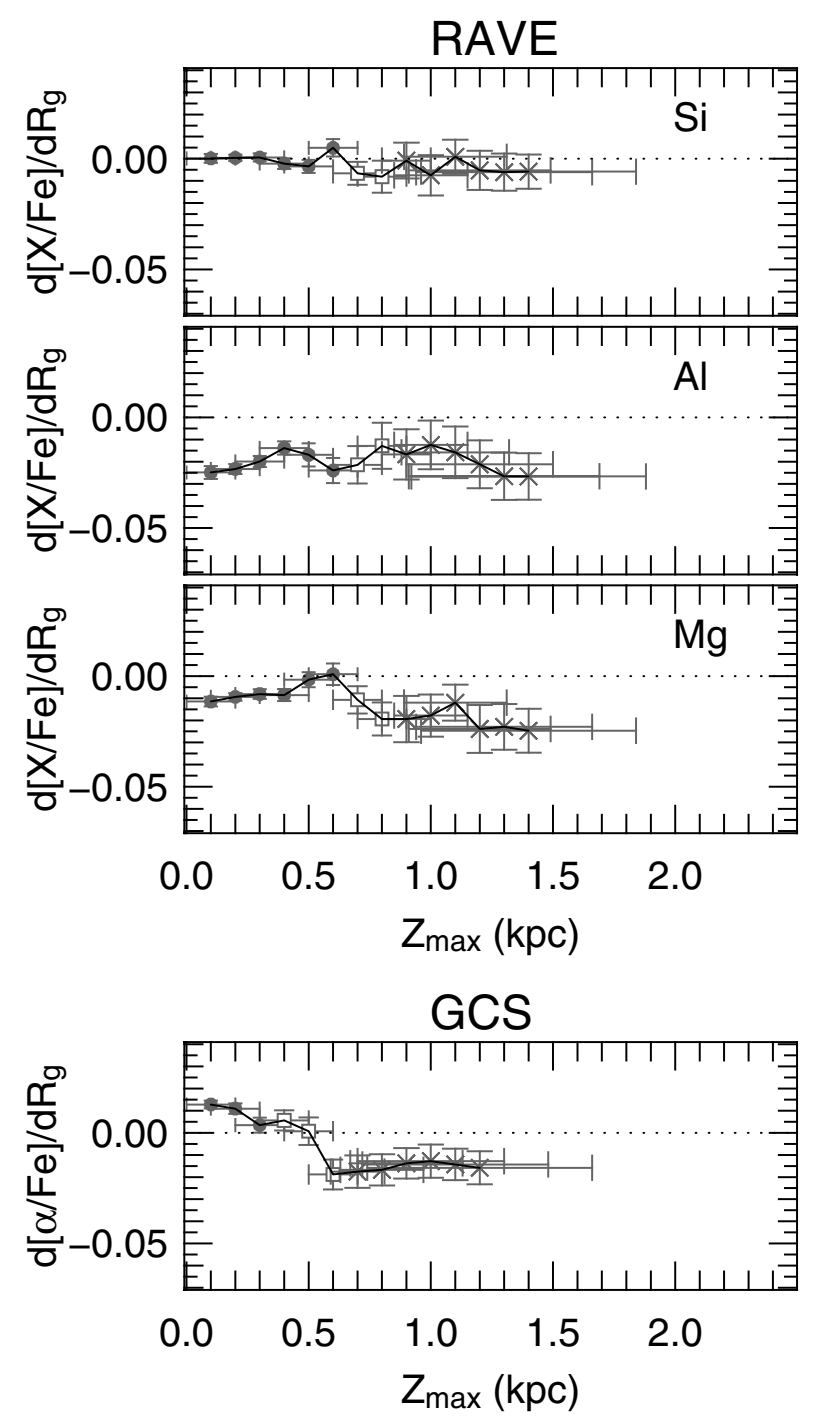

Fig. 6. Gradients for the RAVE relative abundances $[\mathrm{Mg} / \mathrm{Fe}],[\mathrm{Al} / \mathrm{Fe}]$, and $[\mathrm{Si} / \mathrm{Fe}]$ for RAVE (top), and $[\alpha / \mathrm{Fe}]$ for GCS (bottom) as a function of $Z_{\max }$. Horizontal and vertical error bars are defined as in Fig. 4.

turns slighly positive at $8-10 \mathrm{kpc}$. Gibson et al. (2013) predicted negative or slightly negative $[\mathrm{O} / \mathrm{Fe}]$ gradients at the solar radius. Both works give predicted gradients close to our findings.

The model by Schönrich \& Binney (2009a) differs from the previous ones by construction. This model assumes that the disc forms simultaneously at all radii (no inside-out formation), with a higher infall rate at smaller radii. This causes a higher star formation rate at small Galactic radii, which generates a negative radial gradient, enhanced at late times because the inward flow of Fe-enriched gas increases the iron abundance (and decreases the ratio $[\alpha / \mathrm{Fe}])$. In addition to these mechanisms, radial migration constantly acts as flattening agent. This model predicts an iron gradient of $\simeq-0.11 \mathrm{dex} / \mathrm{kpc}$ and a positive gradient for $[\alpha / \mathrm{Fe}]$. This last has the opposite sign to the sign predicted by the inside-out formation models and is also opposite to our observational data, which render a shallow $[\mathrm{Mg} / \mathrm{Fe}]$ and $[\mathrm{Si} / \mathrm{Fe}]$ gradient ( -0.01 and 0.00 , respectively). This seems to favour the inside-out formation scenario, but it does not exclude the flattening action of radial migration on the gradients.

The comparison with other recent chemo-dynamical models are not conclusive. The predicted metallicity gradients range from $-0.02 \mathrm{dex} \mathrm{kpc}^{-1}$ (Kobayashi \& Nakasato 2011)
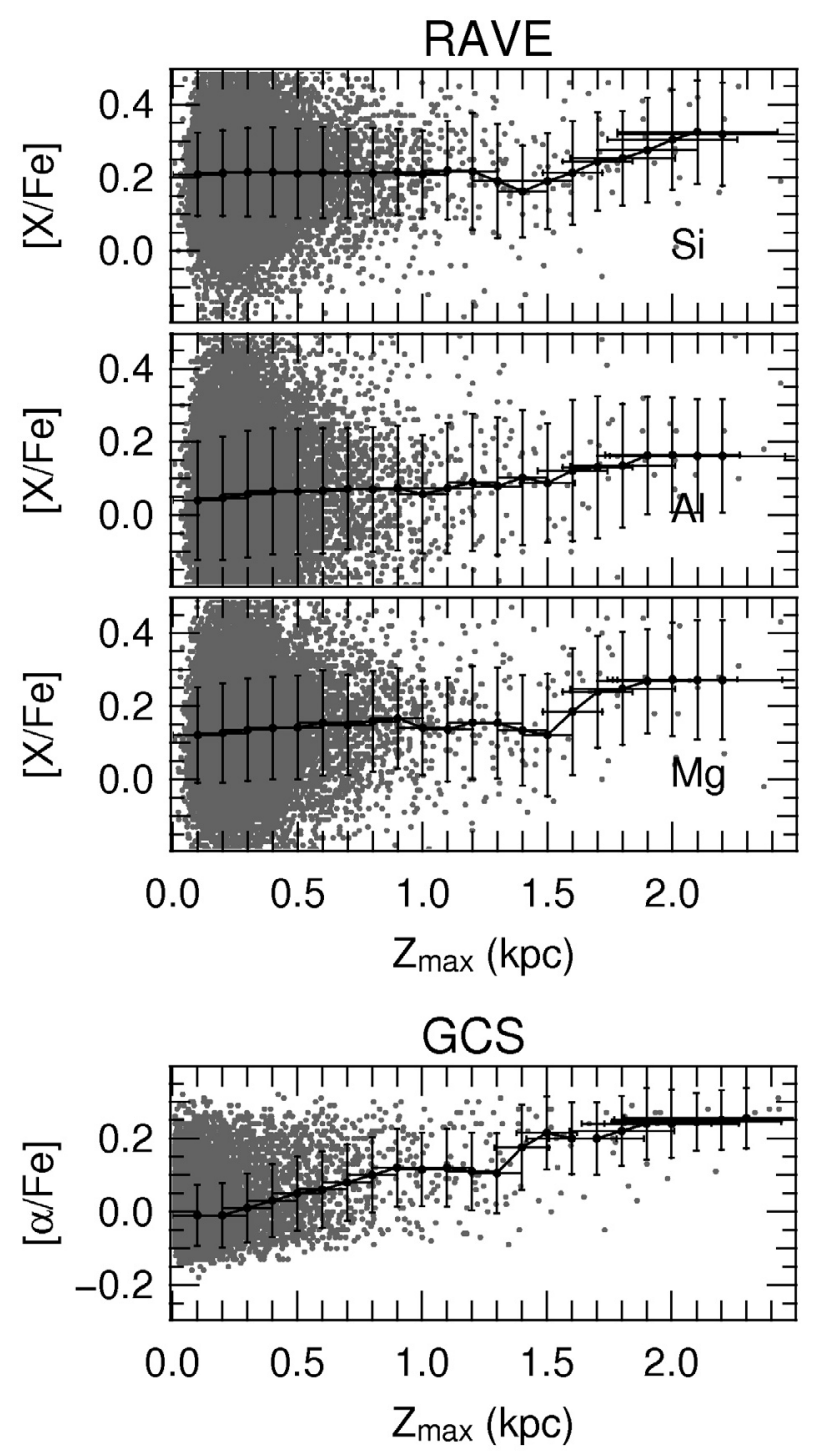

Fig. 7. Relative abundances $[\mathrm{Mg} / \mathrm{Fe}],[\mathrm{Al} / \mathrm{Fe}]$, and $[\mathrm{Si} / \mathrm{Fe}]$ for $\mathrm{RAVE}$ (top), and $[\alpha / \mathrm{Fe}]$ for GCS (bottom) as a function of $Z_{\max }$. Horizontal and vertical error bars are defined as in Fig. 5.

to $-0.06 \mathrm{dex} \mathrm{kpc}^{-1}$ (Minchev et al. 2013). The latter gives a $\mathrm{Fe}$ gradient that closer agrees with our data, whereas the comparison with the $[\mathrm{Mg} / \mathrm{Fe}]$ and $[\mathrm{Si} / \mathrm{Fe}]$ gradient is not possible. Indeed, it is not correct to directly compare the gradients obtained in the present work to those in Fig. 5 of Minchev et al. The reason is that in the RAVE analysis our sample was subdivided into three subsamples as a function of $\mathrm{Zmax}$, hence each of them is composed of stars with a different age mix. As an example, although in Fig. 5 (bottom) of Minchev et al. the predicted [O/Fe] gradients are mostly positive, to compute the gradient for a sample composed of stars with different ages one needs to take in account the density of stars of different age bins. A detailed comparison of the $[\mathrm{Mg} / \mathrm{Fe}]$ and $[\mathrm{Si} / \mathrm{Fe}]$ gradients with the predictions of the chemodynamical models of Minchev et al. will be carried out in a forthcoming paper.

The aluminium abundance gradient deserves a separate discussion. It is much more negative $\left(\sim-0.85 \mathrm{dex} \mathrm{kpc}^{-1}\right)$ than the other elements. The aluminium abundance suffers from non-LTE effects, which depend on the stellar parameters and metallicity (Baumüller \& Gehren 1997). Although the RAVE chemical abundances are based on LTE spectral analysis, non-LTE 
effects are expected to cause mere systematic errors in our Al abundance estimates based on the $\mathrm{Al}$ doublet at $\sim 8773 \AA$ of metal-rich $([\mathrm{m} / \mathrm{H}]>-1.0$ dex $)$ dwarf stars. This has no effects on the gradient determination. Aluminium is also reported to be a useful element to distinguish different stellar populations, such as disc and halo stars (Gehren et al. 2006). Its peculiar gradient may serve as discriminant between different chemical model of the Galaxy. Unfortunately, these element have not had enough attention in the cited chemical evolution studies, and a direct comparison with our findings is not possible.

\section{Conclusions}

We have analysed 19962 dwarf stars selected from the RAVE chemical catalogue and 10616 dwarf stars selected from the GCS making use of chemical, kinematic, and Galactic orbit parameters such as chemical abundances, guiding radii $\left(R_{\mathrm{g}}\right)$, and maximum distance from the Galactic plane reached by the stars along their orbits $\left(Z_{\max }\right)$. The same analyses were also applied to a RAVE mock sample created using the stellar population synthesis code GALAXIA (Sharma et al. 2011), which is based on the Besançon model (Robin et al. 2003). Using the RAVE and GCS sample, we found that the Galactic disc has a negative iron abundance gradient of $-0.065 \mathrm{dex} \mathrm{kpc}^{-1}$ and $-0.043 \mathrm{dex} \mathrm{kpc}^{-1}$ for the RAVE and GCS samples, respectively. We found that for the GCS sample the gradient is more negative $\left(-0.054 \mathrm{dex} \mathrm{kpc}^{-1}\right)$ when stars at small $R_{\mathrm{g}}(<5.5 \mathrm{kpc})$ are excluded from the measurement. This is due to an observational bias that affects volume-limited samples such as the GCS and RAVE: stars from the inner disc can reach the Sun's neighbourhood only if they move on eccentric orbits. Such kinematically hot stars are on average more metal-poor than stars with the same $R_{\mathrm{g}}$, but are on more circular orbits, which will be missing from our sample. This creates a bias against highmetallicity and low- $R_{\mathrm{g}}$ stars, which contributes to make the gradient flatter than it really is. This effect is evident in the GCS sample and barely visible in the RAVE one. To assess the impact of this bias, we measured the gradients with different cuts at low and high $R_{\mathrm{g}}$, with differences in gradients no larger than

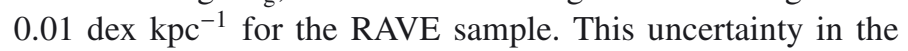
chemical gradients is small enough not to affect our data interpretation. The presence of inhomogeneities in the volumes probed by the RAVE and GCS samples (such as disrupted open clusters and moving groups) cannot be excluded to explain the different obtained gradients. Despite the differences just highlighted, for both RAVE and GCS samples the gradient shows a common trend: it becomes flatter at increasing $Z_{\max }$ (as found by previous studies, e.g. Cheng et al. 2012; Boeche 2011; Pasquali \& Perinotto 1993). The RAVE sample also allows gradient measurements for the elements $\mathrm{Mg}, \mathrm{Al}$, and $\mathrm{Si}$, for which we found the same trend.

The GALAXIA/Besançon mock sample shows striking differences compared with the RAVE and GCS samples, because the iron gradients are positive at any $Z_{\max }$. This surprising and unrealistic result is caused by the lack of correlation between metallicity and tangential velocity (or angular momentum) in the Besançon model. In fact, although the Besançon model assumes a radial gradient of $-0.07 \mathrm{dex} \mathrm{kpc}^{-1}$ for the thin disc (and zero for the other populations), the metallicities are assigned regardless of the kinematics. This means that stars with small $R_{\mathrm{g}}$ have the same probability to be metal-rich as stars with large $R_{\mathrm{g}}$, while in the real Galaxy stars coming from the inner disc are more likely to be metal-rich than stars coming from the outer disc. The absence of a correlation between chemistry and kinematics in the Besançon model removes the metallicity gradient in the mock sample when the kinematically dependent $R_{\mathrm{g}}$ quantity is used. Moreover, the thick-disc stars, with a mean metallicity of -0.78 dex and stronger asymmetric drift than the thin-disc stars, shift to lower $R_{\mathrm{g}}$ and $[\mathrm{Fe} / \mathrm{H}]$, creating a positive metallicity gradient in the full sample even when the individual components have a negative or no radial gradient. The mock sample also shows an excess of thick disc stars compared with the RAVE (and GCS) sample, with a mean metallicity peak at -0.78 dex. This appears too low compared with the RAVE and GCS samples, where a value of -0.5 dex seems more realistic (also suggested by Bilir et al. 2012; Kordopatis et al. 2011b; Schlesinger et al. 2012; Soubiran et al. 2003). The discrepancies between the observational samples and the model can be reduced by i) decreasing the local density; ii) decreasing the vertical velocity dispersion; and iii) raising the mean metallicity of the thick disc.

Our analysis of the gradients as function of $Z_{\max }$ with a moving box car revealed that the abundance gradients remain approximately constant up to $\sim 0.6 \mathrm{kpc}$, and then increase to zero. The change in gradients at $\sim 0.6 \mathrm{kpc}$ does not correspond to a change in average abundance, suggesting that the gradients become flatter because the fraction of kinematically hot, metal-poor (thick-disc) stars become significant at that height and leads to a flatter gradient, as discussed before. Another explanation is that this population of stars has no radial gradient. Both can be true, and our analysis is not able to distinguish between these possibilities. The constancy of the radial gradients up to $Z_{\max } \sim 0.6 \mathrm{kpc}$ reveals that a stellar population with gradient zero (probably the thick disc) does not affect the thin disc properties at these heights and the values obtained here are reliable. For a sample of RAVE stars at $Z_{\max }<0.4 \mathrm{kpc}$ we measured gradients that are steeper $\left(\mathrm{d}[\mathrm{Fe} / \mathrm{H}] / \mathrm{d} R_{\mathrm{g}}=-0.065 \mathrm{dex} \mathrm{kpc}^{-1}\right)$ than predicted by some chemical models (for instance $-0.040 \mathrm{dex} \mathrm{kpc}^{-1}$ by Chiappini et al. 2001, $-0.052 \mathrm{dex} \mathrm{kpc}^{-1}$ by Cescutti et al. 2007) and flatter than others (-0.11 dex kpc ${ }^{-1}$ by Schönrich \& Binney 2009a). We also found slightly negative (or zero) gradients for abundances relative to iron (i.e. $\mathrm{d}[\mathrm{Mg} / \mathrm{Fe}] / \mathrm{d} R_{\mathrm{g}}=-0.009 \mathrm{dex} \mathrm{kpc}^{-1}$ ), which supports the models of Chiappini et al. (2001) and Gibson et al. (2013), and it is in contrast with the model by Schönrich \& Binney (2009a), which predicts positive gradients.

The last model considers radial migration to be an important flattening agent for the radial gradients. Although it is common sense to expect that radial mixing is acting in our Galaxy, it is difficult to estimate the impact of its action on the observed chemical gradients, because migrated stars changed their original $R_{\mathrm{g}}$ (Roškar et al. 2008; Sánchez-Blázquez et al. 2009) and have become kinematically indistinguishable from the local sample. Detailed chemical analyses are therefore necessary to distinguish migrated from the locally born stars. Highresolution and high $\mathrm{S} / \mathrm{N}$ spectroscopic surveys such as the GaiaESO survey (Gilmore et al. 2012), the GALactic Archaeology with HERMES (GALAH) survey (Zucker et al. 2012), and the Apache Point Observatory Galactic Evolution Experiment (APOGEE) survey (Majewski et al. 2010) may be able to identify and quantify migrated stars.

This paper will be followed by a second one in which we measure the radial chemical gradients by using RAVE giant stars. Because RAVE is a magnitude-limited survey, and because giants stars are luminous objects, we are able to cover a larger volume, use the actual Galactocentric distance $R$ in addition to the guiding radius $R_{\mathrm{g}}$, and probe the Galactic disc at larger 
heights. This analysis will provide additional constraints on the chemical and kinematical characteristics of the Milky Way.

Acknowledgements. We acknowledge funding from Sonderforschungsbereich SFB 881 "The Milky Way System" (subproject A5) of the German Research Foundation (DFG). Funding for RAVE has been provided by the Australian Astronomical Observatory; the Leibniz-Institut fuer Astrophysik Potsdam (AIP); the Australian National University; the Australian Research Council; the French National Research Agency; the German Research Foundation (SPP 1177 and SFB 881); the European Research Council (ERC-StG 240271 Galactica); the Istituto Nazionale di Astrofisica at Padova; The Johns Hopkins University; the National Science Foundation of the USA (AST-0908326); the W. M. Keck foundation; Macquarie University; the Netherlands Research School for Astronomy; the Natural Sciences and Engineering Research Council of Canada; the Slovenian Research Agency; the Swiss National Science Foundation; the Science \& Technology Facilities Council of the UK; Opticon; Strasbourg Observatory; and the Universities of Groningen, Heidelberg and Sydney. The RAVE website is at http://www.rave-survey.org.

\section{References}

Allende Prieto, C., Beers, T. C., Wilhelm, R., et al. 2006, ApJ, 636, 804 Andrievsky, S. M., Kovtyukh, V. V., Luck, R. E., et al. 2002, A\&A, 381, 32 Antoja, T., Helmi, A., Bienaymé, O., et al. 2012, MNRAS, 426, L1 Baumüller, D., \& Gehren, T. 1997, A\&A, 325, 1088 Bilir, S., Karaali, S., Ak, S., et al. 2012, MNRAS, 421, 3362 Binney, J. 2010, MNRAS, 401, 2318

Binney, J., Burnett, B., Kordopatis, G., et al. 2013, MNRAS, submitted Boeche, C. 2011, Ph.D. Thesis, urn:nbn:de:kobv:517-opus-52478, Potsdam Universität, http://opus.kobv.de/ubp/volltexte/2011/5247/ Boeche, C., Siebert, A., Williams, M., et al. 2011, AJ, 142, 193 Boeche, C., Chiappini, C., Minchev, I., et al. 2013, A\&A, 553, A19 Breddels, M. A., Smith, M. C., Helmi, A., et al. 2010, A\&A, 511, A90 Burnett, B., Binney, J., Sharma, S., et al. 2011, A\&A, 532, A113

Carraro, G., Geisler, D., Villanova, S., Frinchaboy, P. M., \& Majewski, S. R. 2007, A\&A, 476, 217

Carrell, K., Chen, Y., \& Zhao, G. 2012, AJ, 144, 185

Casagrande, L., Ramírez, I., Meléndez, J., Bessell, M., \& Asplund, M. 2010, A\&A, 512, A54

Casagrande, L., Schönrich, R., Asplund, M., et al. 2011, A\&A, 530, A138

Cescutti, G., Matteucci, F., François, P., \& Chiappini, C. 2007, A\&A, 462, 943

Cheng, J. Y., Rockosi, C. M., Morrison, H. L., et al. 2012, ApJ, 746, 149

Chiappini, C., Matteucci, F., \& Gratton, R. 1997, ApJ, 477, 765

Chiappini, C., Matteucci, F., \& Romano, D. 2001, ApJ, 554, 1044

Colavitti, E., Cescutti, G., Matteucci, F., \& Murante, G. 2009, A\&A, 496, 429

Coşkunoğlu, B., Ak, S., Bilir, S., et al. 2012, MNRAS, 419, 2844

Dehnen, W., \& Binney, J. 1998, MNRAS, 294, 429

Famaey, B., Jorissen, A., Luri, X., et al. 2005, A\&A, 430, 165

Friel, E. D., Janes, K. A., Tavarez, M., et al. 2002, AJ, 124, 2693

Gehren, T., Shi, J. R., Zhang, H. W., Zhao, G., \& Korn, A. J. 2006, A\&A, 451, 1065

Gibson, B. K., Pilkington, K., Brook, C. B., Stinson, G. S., \& Bailin, J. 2013, A\&A, 554, A47

Gilmore, G., Randich, S., Asplund, M., et al. 2012, The Messenger, 147, 25

Górski, K. M., Hivon, E., Banday, A. J., et al. 2005, ApJ, 622, 759

Høg, E., Fabricius, C., Makarov, V. V., et al. 2000, A\&A, 355, L27

Ivezić, Ž., Sesar, B., Jurić, M., et al. 2008, ApJ, 684, 287
Jurić, M., Ivezić, Ž., Brooks, A., et al. 2008, ApJ, 673, 864

Karataş, Y., \& Klement, R. J. 2012, New Astron., 17, 22

Katz, D., Soubiran, C., Cayrel, R., et al. 2011, A\&A, 525, A90

Kobayashi, C., \& Nakasato, N. 2011, ApJ, 729, 16

Kordopatis, G., Recio-Blanco, A., de Laverny, P., et al. 2011a, A\&A, 535, A106

Kordopatis, G., Recio-Blanco, A., de Laverny, P., et al. 2011b, A\&A, 535, A107

Kordopatis, G., Gilmore, G., Steinmetz, M., et al. 2013, AJ, accepted

Lemasle, B., François, P., Piersimoni, A., et al. 2008, A\&A, 490, 613

Luck, R. E., \& Lambert, D. L. 2011, AJ, 142, 136

Luck, R. E., Kovtyukh, V. V., \& Andrievsky, S. M. 2006, AJ, 132, 902

Maciel, W. J., \& Costa, R. D. D. 2010, IAU Symp., 265, 317

Maciel, W. J., \& Quireza, C. 1999, A\&A, 345, 629

Majewski, S. R., Wilson, J. C., Hearty, F., Schiavon, R. R., \& Skrutskie, M. F. 2010, IAU Symp., 265, 480

Matijevič, G., Zwitter, T., Bienaymé, O., et al. 2012, ApJS, 200, 14

Matteucci, F., \& Francois, P. 1989, MNRAS, 239, 885

Minchev, I., Famaey, B., Combes, F., et al. 2011, A\&A, 527, A147

Minchev, I., Chiappini, C., \& Martig, M. 2013, A\&A, 558, A9

Nordström, B., Mayor, M., Andersen, J., et al. 2004, A\&A, 418, 989

Pancino, E., Carrera, R., Rossetti, E., \& Gallart, C. 2010, A\&A, 511, A56

Pasetto, S., Grebel, E. K., Zwitter, T., et al. 2012a, A\&A, 547, A70

Pasetto, S., Grebel, E. K., Zwitter, T., et al. 2012b, A\&A, 547, A71

Pasquali, A., \& Perinotto, M. 1993, A\&A, 280, 581

Pilkington, K., Few, C. G., Gibson, B. K., et al. 2012, A\&A, 540, A56

Robin, A. C., Reylé, C., Derrière, S., \& Picaud, S. 2003, A\&A, 409, 523

Röser, S., Schilbach, E., Schwan, H., et al. 2008, A\&A, 488, 401

Roeser, S., Demleitner, M., \& Schilbach, E. 2010, AJ, 139, 2440

Roškar, R., Debattista, V. P., Quinn, T. R., Stinson, G. S., \& Wadsley, J. 2008, ApJ, 684, L79

Ruchti, G. R., Fulbright, J. P., Wyse, R. F. G., et al. 2011, ApJ, 737, 9

Sánchez-Blázquez, P., Courty, S., Gibson, B. K., \& Brook, C. B. 2009, MNRAS, 398,591

Schlesinger, K. J., Johnson, J. A., Rockosi, C. M., et al. 2012, ApJ, 761, 160

Schönrich, R., \& Binney, J. 2009a, MNRAS, 396, 203

Schönrich, R., \& Binney, J. 2009b, MNRAS, 399, 1145

Sellwood, J. A., \& Binney, J. J. 2002, MNRAS, 336, 785

Sestito, P., Bragaglia, A., Randich, S., et al. 2008, A\&A, 488, 943

Sharma, S., Bland-Hawthorn, J., Johnston, K. V., \& Binney, J. 2011, ApJ, 730, 3

Siebert, A., Williams, M. E. K., Siviero, A., et al. 2011a, AJ, 141, 187

Siebert, A., Famaey, B., Minchev, I., et al. 2011b, MNRAS, 412, 2026

Soubiran, C., Bienaymé, O., Siebert, A. 2003, A\&A, 398, 141

Steinmetz, M., Zwitter, T., Siebert, A., et al. 2006, AJ, 132, 1645

Teuben, P. J. 1995, in Astronomical Data Analysis Software and Systems IV, eds.

R. Shaw, H. E. Payne, \& J. J. E. Hayes, PASP Conf Ser., 77, 398

Veltz, L., Bienaymé, O., Freeman, K. C., et al. 2008, A\&A, 480, 753

Williams, M. E. K., Steinmetz, M., Binney, J., et al. 2013, MNRAS, 436, 101

Wilson, M. L., Helmi, A., Morrison, H. L., et al. 2011, MNRAS, 413, 2235

Yong, D., Carney, B. W., Teixera de Almeida, M. L., \& Pohl, B. L. 2006, AJ, 131,2256

Yong, D., Carney, B. W., \& Friel, E. D. 2012, AJ, 144, 95

Zacharias, N., Urban, S. E., Zacharias, M. I., et al. 2004, AJ, 127, 3043

Zucker, D. B., de Silva, G., Freeman, K., Bland-Hawthorn, J., \& Hermes Team 2012, Galactic Archaeology: Near-Field Cosmology and the Formation of the Milky Way, ASP Conf. Proc., 458, 421

Zwitter, T., Siebert, A., Munari, U., et al. 2008, AJ, 136, 421

Zwitter, T., Matijevič, G., Breddels, M. A., et al. 2010, A\&A, 522, A54 